\title{
Factores asociados a estancia hospitalaria prolongada en neonatos
}

\author{
LUIS ALFONSO MENDOZA T. ${ }^{1}$, MARTHA ARIAS G. ${ }^{2}$, MIGUEL ÁNGEL OSORIO R. ${ }^{3}$ \\ 1. Fundación Hospital San José de Buga. Unidad Central del Valle, Tuluá, Colombia. \\ 2. Fundación Hospital San José de Buga, Colombia. \\ 3. Universidad del Valle, Cali, Colombia.
}

\begin{abstract}
Factors associated with prolonged hospital stay in infants

Objective: To evaluate factors present on newborn admission to a neonatal intensive care and associated with a prolonged hospital stay. Patients and Method: Non-matched case-control study, with 555 infants, 111 with more than 7 days of hospital stay and 444 who stayed hospitalized between 1 and 7 days, between 2005 and 2010. Pre hospitalization maternal factors (age, pregnancy, health insurance, education, prenatal care, marital status, history of preeclampsia, prolonged rupture of membranes, chorioamnionitis infection) and neonatal ones (age at admission, gestational age, birth weight, gender, delivery practice, route of admission, Apgar and type of resuscitation) that were associated with prolonged hospital stay were analyzed. Analyses were conducted using STATA 11.0 and logistic regression in the multivariate analysis. Results: Maternal factors such as prenatal care with less than 5 doctor visits (AOR 2.7, 95\% CI 1.3-5.5), lack of social health insurance (AOR 1.9, 95\% CI 1.4-29), pregnant three or more times (AOR 1.7, 95\% CI 1.1-2.7), neonatal birth weight under 2,000 g (AOR 4.2, 95\% CI 1.9-9.5), need for cardiopulmonary resuscitation (AOR 4.2, 95\% CI 2 - 9.1), gestational age less than 36 weeks (AOR 3.9, 95\% CI 2 - 7.7) and admission to the neonatal unit through emergency room or referral from another hospital (AOR 2.8, 95\% CI 1.7-4.6) were associated with hospital stays longer than 7 days. Conclusions: In-hospital complications that affect a prolonged stay at the health center were social health insurance, maternal education and prenatal care, and these should be considered in the evaluation of the hospital care quality indicators.
\end{abstract}

(Key words: Infant, neonatal intensive care, prolonged hospital stay).

Rev Chil Pediatr 2014; 85 (2): 164-173

\section{RESUMEN}

Objetivo: Evaluar los factores que están presentes al ingreso del recién nacido a una unidad de cuidados intensivos neonatales y que están asociados a una estancia prolongada. Pacientes y Método: Estudio de casos y controles no pareado, con 555 neonatos, 111 con estancia superior a 7 días y 444 con estancia entre 1 y 7

Recibido el 15 de agosto de 2012, devuelto para corregir el 10 de enero de 2013, segunda versión 23 de abril de 2013, tercera versión 22 de julio de 2013, aceptado para publicación el 3 de diciembre de 2013.

Este trabajo cumple con los requisitos sobre consentimiento /asentimiento informado, comité de ética, financiamiento, estudios animales y sobre la ausencia de conflictos de intereses según corresponda.

Correspondencia a:

Luis Alfonso Mendoza Tascón

E-mail: lamendozat@gmail.com 
días, hospitalizados entre los años 2005 y 2010. Se evaluaron factores maternos (edad, gravidez, seguridad social, educación, control prenatal, estado civil, antecedente de preeclampsia, ruptura prolongada de membranas, corioamnionitis, infecciones) y neonatales (edad al ingreso, edad gestacional, peso al nacer, sexo, vía de nacimiento, vía de ingreso, Apgar y tipo de reanimación) previos a la hospitalización que estuvieron asociados con estancias prolongadas. Los análisis se hicieron con STATA ${ }^{\circledR}$ 11.0, empleando la regresión logística en el análisis multivariado. Resultados: Factores maternos como control prenatal con menos de 5 visitas (ORa: 2,7; IC 95\%: 1,3-5,5), carencia de seguridad social en salud (ORa: 1,9; IC 95\%: 1,4-29), gravidez de 3 o más gestaciones (ORa: 1,7; IC 95\%: 1,1-2,7) y neonatales como peso al nacer menor a $2.000 \mathrm{~g}$ (ORa: 4,2; IC 95\%: 1,9-9,5), necesidad de reanimación cardiorespiratoria (ORa: 4,2; IC 95\%: 2-9,1), edad gestacional menor a 36 semanas (ORa: 3,9; IC 95\%: 2-7,7) e ingreso a la unidad neonatal por urgencia o remisión desde otro centro hospitalario (ORa: 2,8; IC 95\%: 1,7-4,6), se hallaron asociados a estancia mayor a 7 días. Conclusiones: Las complicaciones intrahospitalarias que condicionan una estancia prolongada fueron seguridad social en salud, escolaridad materna y control prenatal, y deben ser consideradas en la evaluación de los indicadores de calidad de atención hospitalaria.

(Palabras clave: Neonato, unidad de cuidados intensivos neonatales, estancia prolongada).

Rev Chil Pediatr 2014; 85 (2): 164-173

\section{Introducción}

La estancia hospitalaria es un buen indicador de calidad de la atención en salud ${ }^{1,2}$. La estancia prolongada en una unidad neonatal afecta la calidad, genera costos elevados para el Estado y su familia, afecta el vínculo y condición laboral de los padres, y causa deterioro del estado de salud de los pacientes.

La estancia prolongada en la unidad de cuidados intensivos (UCI) ha sido definida en forma arbitraria ${ }^{3-6}$, oscilando entre 3 a más de 30 días, afectando las incidencias en los diversos estudios, que van desde 5 al 34,2\% $\%^{3-11}$. El tiempo de estancia hospitalaria depende de varios factores, entre los cuales se destacan la co-morbilidad y el grado de severidad de la enfermedad y sus complicaciones ${ }^{12,13}$.

Los cuidados intensivos representan un alto porcentaje del presupuesto de los hospitales, y tiende a aumentar con el tiempo ${ }^{14,15}$. Una UCI puede consumir entre un 22 y $34 \%$ de los costos hospitalarios totales ${ }^{16-19}$. La estancia prolongada (mayor de 13 días) representa el 7,3\% del total de admisiones y consume el 43,5\% de días-cama de una $\mathrm{UCI}^{20}$.

Este tipo de investigaciones son necesarias y útiles para las instituciones de salud dentro del sistema de mejoramiento continuo de la calidad, identificando a uno de los problemas en la calidad de la atención de los neonatos como lo es la estancia prolongada.
Los objetivos de este trabajo fueron evaluar los factores presentes al ingreso del recién nacido a la UCI Neonatal y que están asociados a una estancia prolongada, evaluar el impacto en los costos hospitalarios y describir las principales morbilidades neonatales, en una unidad de cuidados intensivos.

\section{Pacientes y Método}

Estudio caso-control no pareado, en neonatos que ingresaron por riesgo o enfermedad a la UCI neonatal de la Fundación Hospital San José de Buga, centro de referencia para el centro y norte del Valle del Cauca, Colombia entre el 19 de septiembre de 2005 y 18 de febrero de 2010.

La fuente de información fueron los datos extraídos de las historias clínicas materna, obstétrica y neonatal.

Se definió estancia prolongada de un neonato en la UCI neonatal, a la permanencia en días por encima del percentil 75 de la estancia de todos los neonatos que permanecieron hospitalizados y egresaron vivos entre septiembre de 2005 y febrero de 2010. La mediana de estancia fue de 4 días (RI: 3-7 días), por lo cual se decidió considerar la estancia prolongada a toda estancia hospitalaria superior a 7 días.

Se definió como caso a todo neonato expuesto a riesgo de enfermar por causas mater- 
no-obstétricas o con enfermedad que haya sido hospitalizado, con una permanencia superior al percentil 75 de la estancia hospitalaria y que hubiese egresado vivo. Se definió como control todo neonato con riesgo de enfermar o con enfermedad que haya sido hospitalizado, cuya permanencia estuviera por debajo del percentil 75 de la estancia hospitalaria y que hubiese egresado vivo.

Se incluyeron neonatos de 0 a 30 días de edad con riesgo o enfermedad sospechada o confirmada, de cualquier sexo, cuya edad gestacional fuera igual o superior a las 24 semanas, con peso al nacer igual o superior a 500 $\mathrm{g}$ y cuya estancia hospitalaria fuera mayor de un día. Se excluyeron del estudio neonatos que hayan ingresaron a la UCI neonatal para estabilización durante el período de adaptación, cuyo ingreso hubiese sido por ausencia de madre o acudiente, que hayan egresado como remisión a otra institución y que hayan egresado muertos.

La variable resultado se definió como la estancia hospitalaria que superara el tercer cuartil de la estancia en días para todos los neonatos. Las variables independientes maternas incluyeron edad, gravidez, seguridad social en salud, nivel de educación, control prenatal con 5 y más visitas, número de controles prenatales, estado civil, antecedente de preeclampsia, ruptura prolongada de membranas, corioamnionitis, infección urinaria u otro foco infeccioso. Las variables neonatales incluyeron, edad al ingreso en días, edad gestacional al nacer en semanas, peso al nacer en gramos, sexo, vía de nacimiento, vía de ingreso, calificación de Apgar a los 1, 5 y 10 min del nacimiento, reanimación con ventilación a presión positiva (VPP) con ambú y máscara, reanimación con VPP por tubo orotraqueal, realización de masaje cardíaco durante la reanimación y administración medicamentos (adrenalina, bicarbonato, etc.) durante la reanimación.

Los casos y los controles se seleccionaron mediante muestreo aleatorio simple. Con el paquete estadístico Epidat ${ }^{\circledR}$ 3.1, desde una base de datos con 1.987 neonatos egresados desde el 19 de septiembre de 2005, para determinar un odds ratio (OR) de 2 , con proporción de casos expuestos $50 \%$ y proporción de controles expuestos $33 \%$ con una relación de controles por cada caso de 4:1, un nivel de confianza del $95 \%$ y un poder del $80 \%$, se obtuvo un tamaño de muestra de 111 casos y 444 controles.

Análisis estadístico. Los datos fueron trasladados a Stata ${ }^{\circledR}$ versión $11.0 \AA$ para su análisis. Las variables continuas se expresaron como promedios y su desviación estándar o medianas con su rango intercuartil y se evaluaran con la prueba t-test no pareado o Wilcoxon rank-sum, de acuerdo a su distribución para comparar diferencias. Las variables categóricas se expresaron en frecuencias y proporciones y fueron comparadas usando la prueba de $\chi^{2}$ o prueba Exacta de Fisher según correspondiera. Para determinar la asociación entre las variables independientes y estancia prolongada, se calculó el OR con su intervalo de confianza de 95\% (IC 95\%), mientras el análisis multivariado se realizó mediante regresión logística (RL), incluyendo las variables con nivel de significancia $<0,05$ en el análisis bivariado, con probabilidad de retiro mediante el método "hacia atrás" para variables con $\mathrm{p}>0,05$. La RL se aplicó por separado para las variables maternas y las variables neonatales. El modelo final se construyó a través de la metodología descrita por Hosmer y Lemeshow ${ }^{21}$, evaluándose la presencia de colinearidad mediante la estimación de los coeficientes de correlación de Spearman ${ }^{22}$ y la posibilidad de interacción entre variables, como ha sido descrito por Hosmer y Lemeshow ${ }^{21}$. La capacidad de discriminación entre 2 individuos con resultados opuestos se realizó con el área bajo la curva ROC23. Finalmente, se realizó el diagnóstico de la regresión ${ }^{24}$, mediante las pruebas de pruebas de Wald, Hosmer Lemeshow y Likelihoodratio test. El rendimiento del modelo se determinó mediante la sensibilidad, especificidad, valores predictivos positivo y negativo, con sus respectivos IC 95\%. Finalmente, se calculó la capacidad de cada una de las variables del modelo para predecir de manera individual el promedio de estancia y su área bajo ROC. Para todas las pruebas estadísticas se tuvo en cuenta un nivel de probabilidad menor de 0,05 como criterio de significancia.

La investigación careció de conflicto de intereses desde el punto de vista de patrocinios 
o remuneración alguna y fue aprobada por el Comité de Ética institucional.

\section{Resultados}

Para los 555 neonatos, la mediana de la estancia fue de 4 días con rango intercuartil (RI) de 3-7 días, de los cuales 444 tuvieron estancia entre 1 a 7 días y 111 estancia hospitalaria superior a 7 días. La estancia en la UCI neonatal para el grupo que permaneció 7 o menos días tuvo una mediana de 4 (RI: 2-5 días), mientras que para el grupo con estancia de 8 o más días la mediana fue 11 días (RI: 9-17 días).

Entre las variables de riesgo evaluadas, sólo se halló diferencia estadísticamente significativa entre neonatos con y sin estancia de 7 y más días, para gravidez de 3 o más embarazos, carencia de seguridad social en salud, número de visitas durante el control menor a 5 , estado civil soltera, separa o viuda, nivel de educación inferior a 9 años de estudio aprobados, edad gestacional menor a 36 semanas, peso al nacer menor a $2.000 \mathrm{~g}$, vía de ingreso por urgencias o remisión desde otro centro hospitalario, reanimación avanzada con tubo orotraqueal, masaje cardíaco y medicamentos. Para las demás variables no se halló diferencia (tabla 1).

En el análisis bivariado, para las variables maternas, se halló asociación estadísticamente significativa entre estancia superior a 7 días y gravidez de 3 o más embarazos (OR: 1,9; IC 95\%: 1,2-3,1), carencia de seguridad social en salud (OR: 2,; IC 95\%: 1,3-36), número de visitas durante el control menor a 5 (OR: 3,7; IC 95\%: 1,7-7,6), estado civil soltera, separada o viuda (OR: 1,9; IC 95\%: 1,1-3,3) y nivel de

Tabla 1. Variables maternas evaluadas en pacientes de la Unidad Neonatal

\begin{tabular}{|c|c|c|c|c|}
\hline Características & Medida & $\begin{array}{l}\text { Estancia } \\
1 \text { a } 7 \text { días }\end{array}$ & $\begin{array}{l}\text { Estancia } \\
8 \text { y más días }\end{array}$ & $\mathbf{p}$ \\
\hline \multicolumn{5}{|l|}{ Maternas } \\
\hline Edad materna, años & Mediana (RI) & $24(19-30)$ & $23(19-29)$ & 0,386 \\
\hline Carecer de seguridad social en salud & $\mathrm{n}(\%)$ & $66 / 444(14,9)$ & $34 / 111(30,6)$ & $<0,001 *$ \\
\hline Control prenatal con menos de 5 visitas & $\mathrm{n}(\%)$ & $21 / 438(4,8)$ & $17 / 109(15,6)$ & $0,019 *$ \\
\hline Gravidez & Mediana (RI) & $2(1-2)$ & $2(1-3)$ & 0,044 \\
\hline Nivel de educación & Mediana (RI) & $11(5-11)$ & $8,5(5-11)$ & 0,023 \\
\hline Estado civil soltera, separada o viuda & $\mathrm{n}(\%)$ & $55 / 437(12,5)$ & $24 / 109(22)$ & $0,012^{*}$ \\
\hline Antecedente gestacional de preeclampsia & n (\%) & $32 / 436(7,3)$ & $9 / 108(8,3)$ & $0,563^{*}$ \\
\hline Antecedente gestacional de RPM, 18 o más horas & n (\%) & $78 / 436(17,9)$ & $15 / 109(13,8)$ & $0,569 *$ \\
\hline Antecedente gestacional de corioamnionitis & $\mathrm{n}(\%)$ & $9 / 436(2,1)$ & $4 / 107(3,7)$ & $0,310^{*}$ \\
\hline Antecedente gestacional de infección de vías urinarias & n (\%) & $54 / 436(12,4)$ & $15 / 109(13,8)$ & $0,699 *$ \\
\hline \multicolumn{5}{|l|}{ Neonatales } \\
\hline Edad al ingreso, días & Mediana (RI) & $0(0-1)$ & $0(0-2)$ & 0,918 \\
\hline Edad gestacional, semanas & Mediana (RI) & $39(37-40)$ & $37(34-39)$ & $<0,001$ \\
\hline Peso al nacer, $g$ & Mediana (RI) & $3.050(2.700-3.400)$ & $2.600(1.870-3.200)$ & $<0,001$ \\
\hline Peso al nacer inferior al p10 para la EG & $\mathrm{n}(\%)$ & $51 / 444(11,5)$ & $16 / 111(14,4)$ & $0,397^{*}$ \\
\hline Peso al nacer superior al p90 para la EG & n (\%) & $45 / 444(10,1)$ & $11 / 111(9,9)$ & $0,944^{*}$ \\
\hline Sexo masculino & $\mathrm{n}(\%)$ & $250 / 444(56,3)$ & $69 / 111(62,2)$ & $0,264^{*}$ \\
\hline Nacimiento por vagina & $\mathrm{n}(\%)$ & $264 / 444(59,4)$ & $68 / 111(61,3)$ & $0,729 *$ \\
\hline Vía ingreso por urgencias y remisiones & n (\%) & $118 / 444(26,6)$ & $48 / 111(43,2)$ & $<0,001 *$ \\
\hline Apgar $1 \mathrm{~min}$ & Mediana (RI) & $8(7-9)$ & $8(7-8)$ & 0,157 \\
\hline Apgar a $5 \mathrm{~min}$ & Mediana (RI) & $9(7-10)$ & $8(7-9)$ & 0,062 \\
\hline Apgar a $10 \mathrm{~min}$ & Mediana (RI) & $8(8-10)$ & $9(7-10)$ & 0,358 \\
\hline Reanimación $\mathrm{CR}$ con bolsa y máscara & $\mathrm{n}(\%)$ & $62 / 435(14,2)$ & $14 / 109(12,8)$ & $0,704^{*}$ \\
\hline Reanimación CR con VPP con tubo orotraqueal & $\mathrm{n}(\%)$ & $6 / 435(1,4)$ & $12 / 109(11)$ & $<0,001 *$ \\
\hline Reanimación CR con masaje cardíaco & n (\%) & $3 / 436(0,7)$ & $4 / 109(3,7)$ & $0,03^{* *}$ \\
\hline $\begin{array}{l}\text { Reanimación CR con medicamentos (adrenalina, } \\
\text { bicarbonato, etc.) }\end{array}$ & $\mathrm{n}(\%)$ & $14 / 432(3,2)$ & $11 / 107(10,3)$ & $<0,01^{*}$ \\
\hline
\end{tabular}

RI: Rango intercuartil; RPM: Ruptura prolongada de membranas; EG: Edad gestacional; p10: Percentil 10; p90: Percentil 90; CR: cardiorrespiratoria; VPP: Ventilación a presión positiva. ${ }^{*} \chi^{2}$ de Pearson. **Prueba Exacta de Fisher. 
educación inferior a 9 años de estudio aprobados (OR: 2,1; IC 95\%: 1,2-3,8) (tabla 2). Para las variables neonatales, se halló asociación estadísticamente significativa entre estancia superior a 7 días y edad gestacional menor a 36 semanas (OR: 7; IC 95\%: 4,1-12), peso al nacer menor a $2.000 \mathrm{~g}$ (OR: 9,1; IC 95\%: 4,7$17,7)$, vía de ingreso por urgencias o remisión (OR: 2,1; IC 95\%: 1,3-3,3), reanimación cardiorrespiratoria con VPP con tubo orotraqueal (OR: 8,8; IC 95\%: 3-29,3) y reanimación cardiorrespiratoria con administración de medicamentos (OR: 3,4; IC 95\%: 1,4-8,4) y cualquier tipo de reanimación cardiorrespiratoria (OR 3,9; IC 95\%: 1,9-8), mientras la reanimación cardiorrespiratoria con VPP y masaje cardíaco no fue significativo (OR: 5,5; IC 95\%: 0,937,7) (tabla 2).

Después de realizar el análisis multivariado, encontramos que las variables que permanecieron en el modelo fueron para las variables maternas, gravidez de 3 o más embarazos (OR: 1,7; IC 95\%: 1,1-2,7), carencia de seguridad social en salud (OR: 1,9; IC 95\%: 1,4-29) y número de visitas durante el control prenatal menor a 5 (OR: 2,7; IC 95\%: 1,3-5,5), y para las variables neonatales, edad gestacional menor a 36 semanas (OR: 3,9; IC 95\%: 2-7,7), peso al nacer menor a $2.000 \mathrm{~g}$ (OR: 4,2; IC 95\%: 1,9-9,5), vía de ingreso por urgencias o remisión (OR: 2,8; IC 95\%: 1,7-4,6) y cual- quier tipo de reanimación cardiorrespiratoria (OR 4,2; IC 95\%: 2-9,1) (tabla 3).

El modelo clasificó correctamente al $85 \%$ (IC 95\%: 81,9-88,1\%) de los neonatos con y sin riesgo de estancia superior a 7 días, con sensibilidad 37\% (IC 95\%: 27,5-47,6\%), especificidad 96,8\% (IC 95\%: 95-98,6\%), valor predictivo positivo 74,1\% (IC 95\%: 61,5$86,7 \%$ ) y valor predictivo negativo $86,2 \%$ (IC 95\%: 83-89,3\%), mientras que al área bajo ROC del modelo 0,709 (IC 95\%: 0,655$0,763)$. La capacidad predictiva individual de cada una de las variables fue baja, siendo la mejor la edad gestacional menor a 36 semanas (área bajo ROC: 0,655; IC 95\%: 0,608-0,703) y la de menor rendimiento, el control prenatal menor a 5 visitas (área bajo ROC: 0,5554; IC 95\%: 0,518-0,590). Las variables de mayor impacto en el número de días de estancia hospitalaria fueron peso al nacer menor a $2.000 \mathrm{~g}$, edad gestacional al nacer menor a 36 semanas, control prenatal con menos de 5 visitas y carencia de seguridad social en salud (tabla 4).

Entre las variables maternas se halló colinearidad estadísticamente significativa de carencia de seguridad social en salud con escolaridad materna menor a 9 años de estudio aprobados $(p<0,001)$, con estado civil soltera, separada o viuda $(\mathrm{p}<0,001)$ y con control prenatal con menos de 5 visitas ( $\mathrm{p}<0,001)$. También hallamos colinearidad estadísticamente

Tabla 2. Razón de oportunidad (OR, Odds Ratio), intervalos de confianza de $95 \%$ y valor p, de las variables maternas y neonatales asociadas a estancia prolongada

\begin{tabular}{|c|c|c|c|}
\hline Variables & OR & IC $95 \%$ & $\mathbf{p}$ \\
\hline \multicolumn{4}{|l|}{ Variables maternas } \\
\hline Gravidez 3 o más embarazos & 1,9 & $1,2-3,1$ & $<0,01$ \\
\hline Carecer de seguridad social en salud & 2,2 & $1,3-36$ & $<0,001$ \\
\hline Control prenatal con menos de 5 visitas & 3,7 & $1,7-7,6$ & $<0,001$ \\
\hline Estado civil soltera, separada o viuda & 1,9 & $1,1-3,3$ & 0,021 \\
\hline Nivel de educación con menos 9 años de estudio aprobados & 2,1 & $1,2-3,8$ & $<0,01$ \\
\hline \multicolumn{4}{|l|}{ Variables neonatales } \\
\hline Edad gestacional menor a 36 semanas & 7 & $4,1-12$ & $<0,001$ \\
\hline Peso al nacer inferior a $2.000 \mathrm{~g}$ & 9,1 & $4,7-17,7$ & $<0,001$ \\
\hline Vía de ingreso por urgencias o remitido & 2,1 & $1,3-3,3$ & $<0,001$ \\
\hline Reanimación CR con VPP con tubo orotraqueal & 8,8 & $3-29,3$ & $<0,001$ \\
\hline Reanimación CR con VPP y masaje cardíaco & 5,5 & $0,9-37,9$ & 0,01 \\
\hline Reanimación con medicamentos (adrenalina, bicarbonato, etc.) & 3,4 & $1,4-8,4$ & $<0,001$ \\
\hline Necesidad de reanimación cardiorrespiratoria & 3,9 & $1,9-8$ & $<0,001$ \\
\hline
\end{tabular}

OR: Odds ratio; IC 95\%: Intervalo de confianza de 95\%; CR: cardiorrespiratoria; VPP: ventilación a presión positiva. 
Tabla 3. Análisis multivariado y área bajo ROC de cada variable

\begin{tabular}{|c|c|c|c|c|c|c|c|c|}
\hline Variables & ORc & IC $95 \%$ & $\mathbf{P}$ & ORa & IC $95 \%$ & $\mathbf{p}$ & $\begin{array}{l}\text { Área bajo } \\
\text { ROC }\end{array}$ & IC $95 \%$ \\
\hline \multicolumn{9}{|l|}{ Variables maternas } \\
\hline Gravidez 3 o más embarazos & 1,9 & $1,2-3,1$ & $<0,01$ & 1,7 & $1,1-2,7$ & 0,028 & 0,566 & $0,517-0,616$ \\
\hline Carecer de seguridad social en salud & 2,2 & $1,3-36$ & $<0,001$ & 1,9 & $1,4-29$ & 0,017 & 0,600 & $0,523-0,616$ \\
\hline Control prenatal con menos de 5 visitas & 3,7 & $1,7-7,6$ & $<0,001$ & 2,7 & $1,3-5,5$ & $<0,01$ & 0,554 & $0,518-0,590$ \\
\hline Estado civil soltera, separada o viuda & 1,9 & $1,1-3,3$ & 0,021 & 1 & $0,4-2,2$ & 0,912 & & \\
\hline $\begin{array}{l}\text { Nivel de educación con menos } 9 \text { años } \\
\text { de estudio aprobados }\end{array}$ & 2,1 & $1,2-3,8$ & $<0,01$ & 1,4 & $0,7-2,6$ & 0,321 & & \\
\hline \multicolumn{9}{|l|}{ Variables neonatales } \\
\hline Edad gestacional menor a 36 semanas & 7 & $4,1-12$ & $<0,001$ & 3,9 & $2-7,7$ & $<0,001$ & 0,655 & $0,608-0,703$ \\
\hline Peso al nacer inferior a $2.000 \mathrm{~g}$ & 9,1 & $4,7-17,7$ & $<0,001$ & 4,2 & $1,9-9,5$ & 0,001 & 0,623 & $0,579-0,666$ \\
\hline Vía de ingreso por urgencias o remitido & 2,1 & $1,3-3,3$ & $<0,001$ & 2,8 & $1,7-4,6$ & $<0,001$ & 0,583 & $0,533-0,634$ \\
\hline $\begin{array}{l}\text { Necesidad de reanimación } \\
\text { cardiorrespiratoria }\end{array}$ & 3,9 & $1,9-8$ & $<0,001$ & 4,2 & $2-9,1$ & $<0,001$ & 0,557 & $0,522-0,593$ \\
\hline
\end{tabular}

ORc: Razón de oportunidad cruda; ORa: Razón de oportunidad ajustada; IC 95\%: Intervalo de confianza de 95\%.

Tabla 4. Impacto de cada variable predictiva sobre los días de estancia hospitalaria*

\begin{tabular}{|c|c|c|}
\hline Variables & $\begin{array}{c}\text { Estancia en días ( } \pm \text { DE) } \\
\text { Con la variable }\end{array}$ & $\begin{array}{c}\text { Estancia en días ( } \pm \text { DE) } \\
\text { Sin la variable }\end{array}$ \\
\hline \multicolumn{3}{|l|}{ Variables maternas } \\
\hline Gravidez 3 o más embarazos & $7,4( \pm 10,3)$ & $5,7( \pm 7,1)$ \\
\hline Carecer de seguridad social en salud & $9,6( \pm 14,9)$ & $5,3( \pm 5,2)$ \\
\hline Control prenatal con 5 o menos visitas & $10,9( \pm 16,2)$ & $5,8( \pm 7)$ \\
\hline \multicolumn{3}{|l|}{ Variables neonatales } \\
\hline Edad gestacional menor a 36 semanas & $13( \pm 14,3)$ & $5( \pm 5,9)$ \\
\hline Peso al nacer inferior a $2.000 \mathrm{~g}$ & $16,4( \pm 16,9)$ & $5,1( \pm 5,9)$ \\
\hline Vía de ingreso por urgencias o remitido & $6,8( \pm 9,6)$ & $5,9( \pm 7,6)$ \\
\hline Necesidad de reanimación cardiorrespiratoria & $8,9( \pm 7,1)$ & $6( \pm 8,3)$ \\
\hline
\end{tabular}

*Se empleó promedio y desviación estándar.

Tabla 5. Primeros 10 diagnósticos o grupo de diagnósticos que afectaron a los neonatos

\begin{tabular}{|lcccccc|}
\hline Diagnóstico o grupo de diagnósticos & $\begin{array}{c}\text { Estancia } \\
\mathbf{1} \text { a } \mathbf{7} \text { días }\end{array}$ & $\mathbf{\%}$ & $\begin{array}{c}\text { Estancia } \\
\mathbf{8} \text { más días }\end{array}$ & $\mathbf{\%}$ & Total & \% \\
\hline 1 Riesgo o sospecha de infección bacteriana & 171 & 38,5 & 30 & 27 & 201 & 36,2 \\
\hline 2 Taquipnea transitoria & 82 & 18,5 & 14 & 12,6 & 96 & 17,3 \\
\hline 3 Ictericia & 80 & 18 & 3 & 2,7 & 83 & 15,0 \\
\hline 4 Otras dificultades respiratorias & 28 & 6,3 & 9 & 8,1 & 37 & 6,7 \\
\hline 5 Prematuro $\leq$ 34 semanas y sus complicaciones & 5 & 1,1 & 14 & 12,6 & 19 & 3,4 \\
\hline 6 Vómito y otros trastornos alimentarios & 15 & 3,4 & 2 & 1,8 & 17 & 3,1 \\
\hline 7 Asfixia perinatal & 9 & 2 & 7 & 6,3 & 16 & 2,9 \\
\hline 8 Bajo peso para la edad gestacional & 10 & 2,3 & 5 & 4,5 & 15 & 2,7 \\
\hline 9 Otras infecciones bacterianas & 5 & 1,1 & 9 & 8,1 & 14 & 2,5 \\
\hline 10 Neumonía & 6 & 1,4 & 5 & 4,5 & 11 & 2,0 \\
\hline
\end{tabular}


significativa de gravidez de 3 o más embarazos con escolaridad materna menor a 9 años aprobados $(\mathrm{p}<0,001)$, con control prenatal con menos de 5 visitas $(\mathrm{p}<0,001)$, con escolaridad materna menor a 9 años de estudio aprobados, con estado civil soltera, separada o viuda $(\mathrm{p}<$ $0,001)$ y con control prenatal con menos de 5 visitas $(\mathrm{p}<0,001)$. Entre las variables neonatales se halló colinearidad estadísticamente significativa de edad gestacional al nacer menor a 36 semanas con peso al nacer inferior a 2.000 $\mathrm{g}(\mathrm{p}<0,001)$ y con necesidad de reanimación cardiorrespiratoria $(p=0,047)$.

Diagnósticos neonatales. En cuanto a los diagnósticos neonatales, la tabla 5 muestra los principales 10 diagnósticos o grupos de diagnósticos de los neonatos, siendo los principales el riesgo o sospecha de infección bacteriana, taquipnea transitoria, ictericia, otras dificultades respiratorias y la prematuridad 34 o menos semanas con sus complicaciones, que representan el 78,6\% de los diagnósticos.

Las infecciones asociadas a la atención en salud (IAAS) se presentaron en ambos grupos. Aunque no era el objetivo de investigación, las IAAS estuvieron asociadas con la estancia mayor a 7 días (OR: 43,8; IC95\%: 6,1-1902; p < $0,001)$.

Estancia prolongada y costos. Para evaluar los costos generados por estancia prolongada evaluamos el número de días que los neonatos en promedio permanecieron en cuidados intensivos, cuidados intermedios y cuidados básicos. La mediana de los costos para un neonato cuya estancia no superó los 7 días fue U\$300 (RI: U\$210-490), mientras que para aquellos neonatos cuya estancia fue igual o superior a 8 días la mediana del costo de U\$1295 (RI: U\$760-4.700), con una diferencia estadísticamente significativa $(p<0,05)$.

\section{Discusión}

La definición de estancia prolongada en cualquier servicio hospitalario está lejos de ser universal, empleándose definiciones ambigüas como por ejemplo, $5 \%$ de las estancias más $\operatorname{largas}^{25}$ o egreso después de las 42 semanas de edad gestacional corregida para neonatos de menos de 28 semanas $^{12}$.
Varios determinantes influencian la salud de la mujer embarazada y con ello los resultados sobre sus neonatos. El control prenatal modifica sustancialmente los resultados neonatales y maternos, pero aún existen criterios divergentes acerca de cuál debe ser el modelo adecuado del mismo. Se observa muy poca consistencia entre países en términos del contenido de las guías de cuidado prenatal, sugiriendo la necesidad de reexaminar sus contenidos y las evidencias en las que esas recomendaciones se han basado ${ }^{26}$. Hallamos variables maternas asociadas a estancia superior a 7 días, como control prenatal con menos de 5 visitas, gravidez de 3 o más embarazos, carencia de seguridad social en salud, estado civil sin compañero o pareja estable (soltera, separada o viuda) y nivel de educación inferior a 9 años de estudio aprobados. No hallamos que la edad materna y el antecedente de condiciones durante la gestación, como preeclampsia, ruptura prolongada de membranas, corioamnionitis o infección urinaria materna, se asociaran a estancia superior a 7 días. Bellani $\mathrm{P}$ y col. ${ }^{27}$, hallaron que la edad materna inferior a 17 años (riesgo relativo -RR-: 2,54; IC 95\%: 1,28-5,03) y la falta de control del embarazo (RR: 2,76; IC 95\%: $1,44-5,3)$, se asociaron a estancia prolongada (mayor a 60 días).

Pero quizás, las variables que podrían afectar más la estancia hospitalaria son la edad gestacional y el peso al nacer. Hallamos que la edad gestacional inferior a 36 semanas, peso al nacer menor a $2.000 \mathrm{~g}$ y necesidad de reanimación cardiorrespiratoria, se asociaron a una estancia hospitalaria mayor a 7 días. Sin embargo, la restricción del crecimiento intrauterino (peso por debajo del percentil 10 para la edad gestacional) o el nacer con peso grande para la edad gestacional (peso por encima del percentil 90 para la edad gestacional) no se asociaron a una estancia superior a 7 días, como tampoco el sexo, vía de nacimiento, Apgar a $\operatorname{los} 1,5$ y $10 \mathrm{~min}$. Algunos de estos hallazgos son diferentes a los de Korvenranta $\mathrm{E}$ y $\mathrm{col}^{28}$, para quienes el sexo masculino, bajo peso al nacer, peso grande para la edad gestacional y Apgar al primer minuto estuvieron asociados con estancia prolongada. Bellani $\mathrm{P}$ y col. ${ }^{27}$, hallaron asociación entre estancia prolongada y 
peso al nacer inferior a $1.500 \mathrm{~g}$ (RR: 3,39; IC 95\%: 1,83-6,27) y menor edad al ingreso (RR: 2,92; IC 95\%: 1,54-4,69). Cotten CM y cols ${ }^{12}$, encontraron que la prematuridad, sobre todo la extrema, está asociada a morbilidades que prolongan la estancia hospitalaria. Hay patologías y complicaciones como la enterocolitis necrotizante, una complicación del neonato prematuro que requiere intervención quirúrgica (OR: 13,8; IC 95\%: 8,1-23,8), enfermedad pulmonar crónica (OR: 6,8; IC 95\%: 5-9), al menos un episodio de sepsis de comienzo tardío (OR: 1,5; IC 95\%: 1-2,1), más de 2 episodios de sepsis de comienzo tardío (OR: 2,4; IC 95\%: $1,7-3,4)$ y retinopatía de la prematuridad IV/V (OR: 1,5; IC 95\%: 1,1-2) que están asociado a estancia prolongada. Para otros autores, factores previos al ingreso a una unidad neonatal como, transporte neonatal inadecuado (RR: 2,7; IC 95\%: 1,49-4,9), se hallan asociados con una estancia prolongada ${ }^{27}$.

Nuestro trabajo halló que los neonatos que ingresaron por urgencias y los remitidos desde otras instituciones, permanecieron internados más de 7 días. La vía de ingreso del neonato como un estimador de riesgo, refleja la calidad de la atención inicial que reciben los pacientes, la cual puede determinar las complicaciones y con ello la estancia hospitalaria prolongada. Este trabajo no se ocupó de identificar factores relacionados con la organización de los cuidados de la madre y el neonato que pudieran influenciar la estancia prolongada. Sin embargo, al igual que el trabajo de Korvenranta E y $\mathrm{col}^{28}$, los neonatos nacidos en la misma institución y hospitalizados allí mismo, tuvieron una estancia más corta, que los neonatos remitidos desde otras instituciones, argumentando que estas variaciones en los días de hospitalización obedecen a diferentes criterios de egreso de las salas de puerperio, a la comunicación efectiva con los padres para continuar con el proceso de adaptación neonatal en casa y a variaciones en los estilos de manejo neonatal.

Causas diferentes a la prematuridad y bajo peso al nacer, fueron también motivo de hospitalización y a su vez de estancia superior a 7 días. Para Alkiaat A y cols ${ }^{29}$, las causas más frecuentes de hospitalización en un grupo de 1.671 neonatos de termino, fueron las com- plicaciones respiratorias $(25,2 \%)$. La sepsis en el período neonatal también es una causa frecuente de morbilidad y la principal de mortalidad en este grupo de edad, afectando a 30 de cada 1.000 nacidos vivos. Las patologías que más afectaron a nuestros neonatos fueron las infecciosas, las respiratorias, la ictericia y la prematuridad y sus complicaciones. Dentro de la complicaciones en neonatos que son hospitalizados en una UCI, encontramos que las IAAS representan un problema importante en el mundo, como lo demuestra la información registrada por el Centro para la Prevención y el Control de las Enfermedades (CDC) de Estados Unidos de América (EUA), además de otros datos publicados en países como México, Canadá y Brasil, donde se notifican tasas de IAAS que van desde $3 \%$ hasta $25 \%$ de los egresos hospitalarios ${ }^{31,32}$. En nuestro trabajo, el $1,8 \%(10 / 555)$ de los neonatos presentaron alguna IAAS, siendo los menores de 35 semanas (60\%) y menores de 2.000 g (50\%) los más afectados. Aunque nuestro trabajo no se ocupó tampoco de las IAAS, estas están asociadas (RR: 6,26; IC 95\%: 3,52-11,13) a prolongación de la estancia hospitalaria ${ }^{27}$. En un estudio sobre costo-eficacia realizado en EUA se observó que la estancia hospitalaria por IAAS varía de 4 a 68 días, lo que genera gastos que van de 1.833 a 14.626 dólares por cada IAAS ${ }^{33}$. De igual manera, Wenzel RP y col${ }^{34}$, registraron un promedio anual de más de dos millones de IAAS ( $5 \%$ de los egresos hospitalarios), lo que representó un impacto económico de 5 a 10 billones de dólares al año. Goldmann y coll $^{35}$, realizaron un estudio de costos en la UCI neonatal de un hospital en Boston, donde $12 \%$ de los niños admitidos desarrollaron una IAAS, y encontraron un promedio de 10 a 13,8 días por sobre-estancia hospitalaria. Payne NR y col${ }^{36}$, informan que el costo promedio observado para los neonatos de extremado bajo peso que sobrevivieron con alguna sepsis, excedieron a los que no se infectaron en más de dos veces (US\$ 104.473 vs US\$ 49.934). En nuestro trabajo, los pacientes cuya estancia no superó los 7 días tuvieron un costo menor que aquellos con estancias de 8 y más días, diferencia que fue estadísticamente significativa entre los dos grupos. 
Adicional al incremento de los costos, una hospitalización prolongada tiene también otras desventajas como exposición neonatal a estímulos ambientales adversos ${ }^{37,38}$ e impedir el adecuado desarrollo de la relación de los padres con el neonato ${ }^{39}$.

La mayoría de los trabajos publicados hacen referencia a factores neonatales como la prematuridad, el bajo peso al nacer, las patologías y las complicaciones intrahospitalarias, como los principales causantes de estancias intrahospitalarias prolongadas en unidades neonatales. Este trabajo busco factores previos al ingreso a la UCI neonatal, que se halla asociado a una estancia prolongada. Los trabajadores de la salud en todos los niveles, deben identificar aquellos grupos de gestantes cuyos riesgos se traducen en morbilidad materna y neonatal, nacimientos prematuros y de bajo peso al nacer, o con riesgos o patologías, que se convierten en hospitalizaciones y estancias prolongadas.

Este estudio epidemiológico contribuye al análisis de uno de los más graves problemas en la calidad de atención de los pacientes, como lo es la estancia hospitalaria prolongada. Si bien es cierto, la validez de este estudio se restringe a la población neonatal que consultó y fue hospitalizada en la UCI neonatal de la Fundación Hospital San José de Buga, Colombia, la estancia hospitalaria debe ser monitorizada y evaluada como trazador de calidad en las instituciones prestadoras de servicios de salud, así como en los Servicios de Salud local, regional y nacional.

\section{Referencias}

1.- O'Keefe G, Jurkovich GJ, Maier RV: Defining excess resorce utilization and identifying associated factors for trauma victims. J Trauma 1999; 46 (3): 473-8.

2.- Roemer M: Disponibilidad de camas y utilización de los hospitales: un experimento natural. ln: White K, Frenk J, Ordoñez C, Paganini JM, Starfield B, Editor. Investigaciones sobre servicios de salud: una antología. Washington, USA. Organización Panamericana de la Salud 1992: 231-9.

3.- Swoboda S, Dickerson J, Mooney T, et al: Family impact after prolonged surgical intensive care unit stay
(SICU). Crit Care Med 1998; 26 (Suppl): A38.

4.- Goldhill DR, Sumner A: Outcome of intensive care patients in a group of British Intensive Care Units. Crit Care Med 1998; 26: 337-45.

5.- Weissman C, Sprung CL: The impact of long term patients on surgical ICU bed utilization: Year-to-year variation. Crit Care Med 1999; 27 (Suppl): A155.

6.- Niskanen M, Puokonen, Takala J, et al: Quality of life after prolonged intensive care. Crit Care Med 1999; 27: 1132-9.

7.- Rapoport J, Teres D, Lemeshow S, et al: Explaining variability of cost using a severity-of-illness measure for ICU patients. Med Care 1990; 28: 338-48.

8.- Oye RK, Bellamy PE: Patterns of resource consumption in medical intensive care. Chest 1991; 99: 685-9.

9.- Wong DT, Gómez M, McGuire GP, et al: Utilization of intensive care unit days in a Canadian medical-surgical intensive care unit. Crit Care Med 1999; 27: 1319-24.

10.- Richter JC, Pajonk FG, Waydhas Ch: Prolonge surgical ICU treatment: Quality of life as an outcome criterium. Crit Care Med 1999; 27 (Suppl): A159.

11.- Bashour CA, Yared JP, Ryan TA, et al: Long-term survival and functional capacity in cardiac surgery patients after prolonged intensive care. Crit Care Med 2000; 28: 3847-53.

12.- Cotten CM, Oh W, McDonald S, et al: Prolonged Hospital Stay for Extremely Premature Infants: Risk Factors Center Differences, and the Impacto f Mortality on Selecting a Best-Performing Center. J Perinatol 2005; 25: $650-5$.

13.- Progreso para la infancia: Un mundo apropiado para los niños y las niñas. UNICEF 2007 (acceso 2 de febrero de 2012). Objetivo de un mundo apropiado para los niños: Reducir la tasa del bajo peso al nacer por lo menos en un tercio. Disponible en: http://www.unicef. org/spanish/progressforchildren/2007n6/index_41508. htm.

14.- Ruttimann UE, Patel KM, Pollack MM: Length of stay and efficiency in pediatric intensive care units. J Pediatrics 1998; 1: 79-85.

15.- Gemke RJ, Bonsel GJ, Van Vught AJ: Effectiveness and efficiency of a Dutch pediatric intensive care unit: Validity and application of the Pediatric Risk of Mortality score. Crit Care Med 1994; 9: 1477-84.

16.- Halpern NA, Bettes L, Greenstein R: Federal and nationawide intensive care unit and healthcare costs: 19861992. Crit Care Med 1994; 22: 2001-7.

17.- Chalfin DB, Cohen IL, Lambrinos J: The economics and cost effectiveness of critical care medicine. Intensive Care Med 1995 Nov; 21 (11): 952-61. 
18.- Fuchs VR: No pain, no gain: perspectives on cost containment. N EngI J Med 1993; 269: 631-3.

19.- Pauly $M V$ : When does curbing health costs really help the economy? Health Aff (Millwood) 1995; 14: 68-82.

20.- Wong DT, Gómez M, McGuire GP, et al: Utilization of intensive care unit days in a Canadian medical-surgical intensive care unit. Crit Care Med 1999; 27: 1319-24.

21.- Hosmer DW, Lemeshow S: Applied logistic regression. 2nd ed. New York: John Wiley \& Sons; 2000.

22.- Salinas M: Modelos de regresión y correlación IV. Correlación de Spearman. Cienc Trab 2007; 9 (25): 143-5.

23.- Hanley JA, McNeil BJ: The meaning and use of the area under the receiver operating characteristic (ROC) curve. Radiology 1982; 143: 29-36.

24.- Servicio de Medicina Interna, Hospital Punta de Europa, Algeciras, Cádiz (sede Web)*. Algeciras, Cádiz: Sociedad Andaluza de Enfermedades Infecciosas; 29/05/08 (acceso 27 de noviembre de 2008). De Fco. Javier Caballero Granado. Modelos de regresión logística incondicional (II). Disponible en: http://saei.org/ hemero/epidemiol/nota5.html.

25.- Weissman Ch: Analyzing ICU census data: Identifying long-term patients. Crit Care Med 1999; 27 (Suppl): A46.

26.- Casini S, Lucero G, Hertz M, Andina E: Guía de control prenatal. Embarazo normal. Rev Hosp Mat Inf Ramón Sardá 2002; 21 (2): 51-62.

27.- Bellani P, de Sarasqueta P: Factores de riesgo de morbilidad neonatal, internación prolongada y predictors de discapacidad future en una unidad de cuidados intensivos neonatales de alta complejidad. Arch argent pediatr 2005; 103 (3): 218-23.

28.- Korvenranta E, Linna M, Häkkinen $U$, et al: Differences in the length of inicial hospital stay in very preterm infants. Acta Paediatrica 2007; 96: 1416-20.

29.- Alkiaat A, Hutchinson M, Jacques A, Sharp MJ: Evaluation of the frequency and obstetric risk factors associated with neonatal admissions to special care units. ANZJOG 2013; DOI: 10.1111/ajo.12070. Disponible en: http://onlinelibrary.wiley.com/doi/10.1111/ ajo.12070/full.

30.- Jeeva S, Ramesh A, Ashok KD, Vinod KP: Sepsis in the newborn. Indian J Pediatr 2008; 75 (3): 261-6.

31.- Methar $S$ : Setting up a cost-effective programme. En: Mehtar S, ed. Hospital infections control. London: Oxford Medical Publications, 1992: 8-16. 32.- Haley RW, Culver DH, White JW, Morgan WM, Emori TG: The nationwide nosocomial infection rate: A new need for vital statistics. Am J Epidemiol 1985; 121: 159-69.

33.- Chaudhuri AK: Infection control in hospital: Has is quality-enhacing and cost-effective role been apreciated? J Hosp Infect 1993; 25: 1-6.

34.- Wenzel RP: Nosocomial infections, diagnosis related groups and study on the efficacy of nosocomial infection control. Economic implications for hospitals under the prospective payment system. Am J Med 1985; 78 suppl 6B: 3-7.

35.- Goldmann DA, Durbin WA, Freeman J: Nosocomial infections in a neonatal intensive care unit. J Infect Dis 1981; 144: 449-59.

36.- Payne NR, Carpenter JH, Badger GJ, Horbar JD, Rogowski J: Marginal Increase in Cost and Excess Length of Stay Associated With Nosocomial Bloodstream Infections in Surviving Very Low Birth Weight Infants. Pediatrics 2004; 114 (2): 348-55.

37.- Zar $L K$, Balian L: Responses of premature infants to Soutine nursing interventions and Boise in the NIUC. Nurs Res 1995; 44: 179-85.

38.- Rivkees SA, Mayes L, Jacobs H, Gross I: Rest-activity patterns of premature infants are regulated by cycled lighting. Pediatrics 2004; 113: 833-9.

39.- Flacking R, Ewald U, Nyqvist KH, Starrin B: Trusful Bonds: a key to "becoming a mother". And to reciprocal breastfeeding. Stories of mother of very preterm infants at a neonatal unit. Soc Sci Med 2006; 62: 70-80. 